\title{
Archives
}

\section{Whatley, Patricia et Caroline Brown. Archives and Recordkeeping : Theory Into Practice. London : Facet Publishing, 2013, 260 pages}

\section{Alexandra Buthiaux}

Volume 46, numéro 1, 2016

URI : https://id.erudit.org/iderudit/1035725ar

DOI : https://doi.org/10.7202/1035725ar

Aller au sommaire du numéro

Éditeur(s)

Association des archivistes du Québec (AAQ)

ISSN

0044-9423 (imprimé)

2369-9256 (numérique)

Découvrir la revue

Citer ce compte rendu

Buthiaux, A. (2016). Compte rendu de [Whatley, Patricia et Caroline Brown. Archives and Recordkeeping : Theory Into Practice. London : Facet Publishing, 2013, 260 pages]. Archives, 46(1), 101-106. https://doi.org/10.7202/1035725ar

Ce document est protégé par la loi sur le droit d'auteur. L'utilisation des services d'Érudit (y compris la reproduction) est assujettie à sa politique d'utilisation que vous pouvez consulter en ligne.

https://apropos.erudit.org/fr/usagers/politique-dutilisation/
Cet article est diffusé et préservé par Érudit.

Érudit est un consortium interuniversitaire sans but lucratif composé de l’Université de Montréal, l’Université Laval et l’Université du Québec à Montréal. Il a pour mission la promotion et la valorisation de la recherche. https://www.erudit.org/fr/ 


\title{
COMPTE RENDU
}

Whatley, Patricia et Caroline Brown. Archives and Recordkeeping: Theory Into Practice. London: Facet Publishing, 2013, 260 pages.

\author{
Alexandra Buthiaux \\ Archiviste, Ville de Sorel-Tracy
}

\section{RÉSUMÉ}

Ce livre est issu d'un collectif, dont chacun des chapitres a été rédigé par un contributeur distinct. L'introduction rédigée par l'éditrice du collectif, Caroline Brown, met en lumière le fil conducteur de l'ouvrage qui consiste en la revue de la littérature sur la théorie archivistique. L'objectif est de comprendre ses impacts et ses liens avec le monde de la pratique afin que les professionnels de la gestion documentaire puissent s'y intéresser et participer à la recherche. Cette revue ne s'attarde pas à tous les aspects de la gestion des documents et des archives, mais en sélectionne quelques-uns. Elle spécifie que les termes "document" et "archives", ainsi que "archiviste" et "gestionnaire de documents" seront utilisés indifféremment dans le but d'être lus par toutes les personnes responsables des archives et des documents (pour alléger le compte rendu, nous ferons de même).

Caroline Williams met en perspective qu'avec les changements et le développement des technologies, des organisations et des environnements, ainsi qu'avec le développement des médias sociaux (réduction de la frontière entre les professionnels et les non-professionnels), il devient évident que les approches techniques et théoriques doivent évoluer. La compréhension de la nature, de la valeur et du rôle des archives et des documents permet donc de savoir comment se comporter dans ces nouveaux environnements.

Mme Williams met en lumière, en tout premier lieu, que bien que les frontières entre les définitions des rôles et des valeurs des archives soient floues, en fonction de la perspective (un individu, une organisation ou la société), il n'en demeure pas moins qu'elles peuvent en revêtir des différences. Elle développe donc pour chacune de ces perspectives des définitions.

Dans un second temps, elle présente des définitions des termes "document" et "archives", issues de différentes professions, d'organisations, de milieux institutionnels ainsi que des normes internationales, et démontrent qu'elles sont multiples et variées.

Caroline Williams confronte ensuite la théorie à la pratique dans le but de confirmer que l'interaction entre les deux est importante. Ceci est explicité à travers l'exemple du passage du cycle de vie (compréhension plus réaliste du système de gestion documentaire prénumérique) au modèle du continuum qui s'avère plus cohérent dans le milieu dynamique et intangible du numérique. 
Le chapitre 2 a pour thème l'évaluation. Son auteure, Anne Gilliland, introduit l'évaluation en passant en revue la relation des archivistes avec celle-ci, résume quelques propositions et questions émergentes qui ont participé à l'évolution de la pratique archivistique. Elle brosse à la suite une histoire détaillée de l'évaluation. Elle présente les différentes stratégies ou méthodes (Shellenberg and beyond, Minnesota Method, Documentation strategy and macroanalysis, Postcustodiality) qui ont été développées au fil du temps. Elle note que dans le contexte de la numérisation des documents, la question est passée de "comment doit-on faire l'évaluation?" à "devons-nous évaluer le matériel numérique?". L'auteure montre également que l'évaluation pose des problèmes de représentativité : la sous-documentation de certaines identités, la mise au ban de certains documents considérés comme "difficiles" (impopulaires, désagréables ou controversés), l'effet de cette évaluation sur l'environnement, etc. Ainsi, plusieurs questions viennent remettre en cause la pratique de l'évaluation.

Anne Gilliland démontre qu'il y a une diversité de pratiques et d'idées. Elle mentionne que dans les organisations on ne peut attendre de trouver l'approche idéale pour traiter correctement les documents. L'énergie mise pour la sélection des documents nés numériques devrait être transférée dans une autre activité. Les efforts devraient plutôt se porter sur: le développement de stockages plus écologiques, de moyens plus sophistiqués et réfléchis pour décrire et repérer les documents, ainsi que sur la compartimentation de la quantité massive de matériel préservé pour soutenir les besoins d'utilisation et de réutilisation toujours plus vastes des utilisateurs. De plus, elle souligne qu'il est impensable de pouvoir éliminer toutes traces des documents numériques que l'on retrouve sur les réseaux. Elle considère qu'il vaudrait mieux se concentrer à retenir et sécuriser la preuve numérique et que les professionnels deviennent les agents du contrôle intellectuel et physique. La seule élimination sélective envisageable est celle basée sur l'oubli. Pour elle, l'évaluation a fait son temps.

Jennifer Meehan, dans le chapitre 3, traite principalement du travail intellectuel de la disposition et de la description. Dans un premier temps, elle souligne le fait que l'archiviste doit prendre conscience de certains biais générés par différents éléments : son travail subjectif qui impose sa vision des choses; son ordre, devenant ainsi la disposition officielle; la hiérarchisation (classification et taxonomies) qui est l'expression d'un point de vue parmi d'autres; la standardisation de la description des archives qui génère le risque de décrire de la même façon des objets créés dans des contextes différents.

La description participative avec le Web 2.0, permettant de mélanger les différents points de vue, est proposée comme solution. Autre piste: le contexte archivistique, dans lequel les archives sont "construites", doit être documenté et inclus dans l'outil de recherche et dans la documentation fournis aux usagers. Ceux-ci pourront alors avoir une idée du contexte institutionnel des pratiques de la disposition et de la description, et du processus individuel des praticiens.

Un autre défi demeure : l'accès aux archives. Dans l'environnement d'aujourd'hui, si on ne les trouve pas en ligne, pour beaucoup d'utilisateurs, elles n'existent pas. L'auteure propose par exemple des métadonnées, qui peuvent passer dans d'autres systèmes, puisque les usagers ont tendance à lancer leur recherche de Google ou de Wikipédia. L'intégration des outils de navigations sociales dans le système descriptif a le potentiel de permettre aux chercheurs d'interagir avec les archivistes. 
Jennifer Meehan explique l'importance d'ouvrir la description archivistique au partage et au participatif pour la découverte et l'accès dans l'environnement connecté. Elle montre également la nécessité pour la profession de se tourner vers un mode Archives 2.0 de pensée et de pratique ou de crowdsourcing (production participative). La description sera ainsi plus orientée vers l'utilisateur, avec la collaboration de l'archiviste et de l'usager, pour construire l'archive elle-même.

Le chapitre 4 de Jeannette A. Bastian examine la relation de la profession avec l'éthique. La première section est consacrée à la perspective éthique elle-même et au besoin des professionnels d'en posséder une. Une brève revue de la littérature des archives et des documents administratifs explore comment les professionnels se sont efforcés d'établir leur propre perspective éthique. Elle discute ensuite de l'éthique dans la pratique et de l'éventail de problèmes auxquels sont confrontés les gestionnaires de documents et les archivistes face à celle-ci.

La seconde partie considère les problèmes éthiques applicables aux archives courantes du XXI ${ }^{e}$ siècle: l'éthique comme composante de la justice sociale, l'éthique pour les minorités culturelles et l'éthique dans l'environnement numérique.

L'auteure démontre que l'éthique est importante pour que les documents conservent leur intégrité et leur probité, ainsi que pour garantir une relation de confiance avec les utilisateurs des archives. L'archiviste est donc un médiateur de la libre circulation de l'information et peut également être un rempart (lanceur d'alerte) contre les abus de l'organisation, qui peut parfois avoir des objectifs en conflit avec l'éthique.

Eric Ketelaar aborde dans le chapitre suivant les thèmes de la mémoire et de l'identité, les mettant en lien avec la gestion des documents.

La première partie du chapitre est consacrée à la définition de la mémoire et de l'identité ainsi que leurs interactions. Il présente ensuite les différents rôles que les archives possèdent dans la construction de la mémoire, pour ensuite définir les quatre conditions pour que les archives soient des composantes de la mémoire culturelle. Il termine cette première section en définissant les particularités des archives (ce qui font d'elles leur caractère unique) par rapport aux autres textes de mémoire (memory texts).

Dans la seconde partie, Ketelaar s'intéresse à la manière dont les théories et les concepts vus dans la section précédente peuvent influencer les politiques et les pratiques dans la formation et la préservation des documents. Il tente, en même temps, de donner quelques conseils et exemples dans le but d'améliorer le rôle des archives et des archivistes dans la construction et la transmission des mémoires et des identités individuelles et collectives.

Il conclut qu'il est important que les archivistes comprennent et appréhendent les liens entre les archives et la construction de la mémoire collective et individuelle, les identités, ainsi que de la nécessité d'agir dans toutes les dimensions du modèle du continuum. Il ne faut pas penser les archives seulement dans la préservation du passé, mais également comme agissant dans la responsabilisation des causes sociales et de justice, dans la formation d'identités et dans les actions de réconciliation avec le passé.

Les identités sont enracinées dans les mémoires et celles-ci ont besoin d'inscription et d'espace. Inscription et espace seront localisés dans le nuage et maintenus par les individus, les groupes et les institutions de mémoire. Les archivistes 
devront collaborer avec les communautés et les professionnels des autres institutions de mémoire et contribuer aux nouvelles formes du Web 2.0 et à l'archivistique participative, devenant ainsi actif dans la communauté plutôt que d'être uniquement des professionnels derrière les murs de leur propre institution.

Le chapitre 6 de Rachel Hardiman, à contre-courant, reprend le travail des philosophes depuis le XVIII ${ }^{\mathrm{e}}$ siècle afin d'améliorer l'approche conceptuelle et de comprendre d'où proviennent les théories occidentales pour les disciplines, les professions et les pratiques de gestion des archives. Pour chaque théorie philosophique, elle démontre la nature de l'influence de celle-ci sur la théorie archivistique et la manière dont elle s'est exprimée. Elle relève quelques points d'exploration possibles permis par ce survol, souhaitant entraîner ses lecteurs à réfléchir et à mettre en œuvre d'autres solutions de leur propre gré.

Le dernier chapitre s'intéresse, comme dans le premier, à la pratique et aux théories, mais au lieu de traiter de leurs fondements, il s'attarde au présent et au futur.

Alan R. Bell s'intéresse dans un premier temps à l'évolution des tensions entre les changements technologiques et la gestion des documents. Il signale qu'avec l'arrivée des ordinateurs et de l'Internet tout a été modifié, même la manière dont les personnes se souviennent et prennent part à la communauté. Il démontre que les utilisateurs ont de nouvelles interactions avec les archives, interactions qui s'éloignent des processus de contrôle traditionnels de la gestion documentaire: responsabilisation des usagers qui se sentent propriétaires des documents, liberté et autonomie qu'éprouve l'usager, accentuation sur le contenu et non plus sur le contenant.

Dans un second temps, il présente la survivance de l'idée de document à la vague numérique: avec l'ère du WYSIWYG (what you see is what you get), il y a transposition sans heurts des actes (création, classement, etc.) et du vocabulaire du domaine papier au domaine numérique. Cependant avec l'utilisation du Web 2.0 s'effectue un changement dans le contrôle de l'information, du système centralisé vers les individus et d'une diminution de l'importance du contexte et de la structure au profit du contenu et de la fluidité.

Ensuite, Bell aborde et étudie la nouvelle définition de document proposée par l'archiviste américain Geoffrey Yeo publiée dans deux articles de la revue American Archivist et qui définit le document comme une représentation persistante des activités.

Dans une dernière partie, il fait la liste des exagérations et des hyperboles qu'il a utilisées dans ce chapitre pour faciliter l'analyse précédente:

- les changements technologiques représentent un défi pour la profession;

- le Web 2.0 est accepté universellement et est un phénomène démocratisé;

- l'emphase sur la théorie et l'exclusion de la pratique;

- le fait que la perspective de gestion des documents soit occidentale et bureaucratique.

Pour conclure, Bell présente quelques pensées telles que: la gestion des documents et des archives est simplement en train de répondre à une autre étape dans son processus d'évolution; l'archiviste doit reconnaître la nature politique de ses décisions; le rôle de la théorie de la gestion des archives est de réduire la distance entre les utilisateurs, les communautés, le document et le gestionnaire de documents, etc. 


\section{ANALYSE CRITIQUE}

Le livre Archives and Recordkeeping: Theory Into Practice est effectivement, comme il l'est mentionné sur sa quatrième de couverture, une revue des définitions, des théories de la gestion des documents et des archives et de leur évolution. Ces différentes synthèses par thématique permettent de brosser un portrait global de la gestion documentaire, de ses concepts, de ses questionnements et de ses principaux défis.

Bien que chaque sujet soit traité par un auteur différent, il n'en reste pas moins qu'il émane de l'ouvrage une certaine uniformité dans la manière de réfléchir à la gestion des documents et des archives. On trouve plusieurs thématiques qui axent cette réflexion.

Cette dernière est d'abord toujours ramenée aux défis auxquels sont confrontés les archivistes. L'expression utilisée par les auteurs de "pratiquer sur des sables mouvants" correspond au contexte actuel du monde numérique et du Web 2.0. Ce nouvel environnement dynamique et instable impose aux praticiens qui y sont confrontés de nouveaux défis auxquels les auteurs tentent de donner des solutions ou des pistes de solutions. Il s'agit là d'un basculement des pratiques (brouillage des frontières entre le public et le privé, entre l'organisationnel et le personnel, etc.)

Un second axe qui revient également est le caractère subjectif des interventions des professionnels sur les documents et les archives. Il revient à plusieurs reprises que l'archiviste doit se rendre compte de cet état de fait, et cette prise de conscience doit se traduire par des actions documentées et éthiques. Au chapitre de l'évaluation, cet axe en vient même à questionner le bien-fondé de celle-ci, car cet acte posé par l'archiviste est ressenti et décrit comme un acte politique, subjectif. Dans la description et le classement des archives et des documents, cela se traduit également par exemple, par la mise en garde de l'archiviste contre le danger de ne pas être conscient de ces gestes, imposant en quelque sorte son point de vue.

Certains chapitres proposent des solutions plus concrètes alors que certains en restent seulement aux formulations de questions qui se posent avec cette ère de changement. On notera cependant que dans leur démonstration les auteurs tentent de fournir le plus de références et d'exemples possibles pour appuyer celle-ci. Globalement, les propositions concrètes encore éparses semblent prouver que le numérique est un chantier encore en réflexion et en attente de propositions concrètes. Dans les prémisses de propositions, les auteurs soulignent la place centrale de l'archiviste comme élément médiateur. Pont entre les archives et les utilisateurs, acteur construisant et préservant la mémoire en collaboration avec les utilisateurs; pratique orientée vers le Web 2.0 avec une approche participative. L'archiviste doit être connecté à cet environnement en mouvement et doit créer la gestion des archives et des documents 2.0.

Il est également le medium entre les différents professionnels dans l'organisation. L’approche interdisciplinaire est invoquée comme élément nécessaire pour arriver à répondre aux défis de ce nouvel environnement. Pour la gestion du système en lui-même, l'archiviste doit être en mesure de travailler avec les informaticiens, les gestionnaires de connaissances ou encore d'autres corps de métier pour trouver sa place - qu'on ne lui donne pas toujours facilement - au sein des organisations qui 
font face à l'avalanche d'informations et à l'ouverture de ces dernières avec le besoin de transparence.

\section{ÉVALUATION DE L'OUVRAGE}

Cet ouvrage permet d'avoir, en une seule lecture, une synthèse et un survol de l'ensemble des théories, concernant la gestion des documents et des archives, qui ont été développées au cours du siècle dernier à aujourd'hui. Pour quelqu'un qui voudrait prendre un peu de recul avec la pratique et alimenter sa réflexion théorique, l'ouvrage est un bon point de départ. Bien sûr, il sera nécessaire de lire d'autres auteurs, d'avoir d'autres références pour confronter cette vision de la gestion des documents et des archives aussi bien pour l'historique de la théorie que pour les solutions proposées qui sont faites pour les défis présents et futurs. Ceci entre dans la perspective du livre, puisque, dans l'introduction, Caroline Brown souligne le fait qu'avoir des perspectives différentes est enrichissant et que le livre est là pour encourager plus de praticiens à s'engager dans des débats conceptuels et à faire de la recherche.

J'encourage donc les professionnels comme les étudiants à lire cet ouvrage pour venir compléter et cultiver leurs connaissances théoriques en matière de gestion des documents et des archives. 